\title{
Rehabilitation and Return to Play of the Athlete after an Upper Extremity Injury
}

\author{
Sameer R. Oak, M.D., Brooks Klein, P.T., D.P.T., S.C.S., Neil N. Verma, \\ Benjamin Kerzner, B.S., Luc M. Fortier, B.A., Neha S. Chava, \\ Michael M. Reinold, P.T., D.P.T., S.C.S., A.T.C., C.S.C.S., C-P.S., and Asheesh Bedi, M.D.
}

Shoulder and elbow injuries during athletic participation are very common and may require operative intervention if refractory to conservative care. In recovering from these upper extremity injuries, proper postoperative rehabilitation and setting reasonable expectations regarding return to play are very important. This review article focuses on the most common surgically treated shoulder and elbow injuries, including rotator cuff tears, SLAP tears, anterior and posterior shoulder instability, and elbow ulnar collateral ligament tears. Rates of return to play after surgical intervention are encouraging in most professional and recreational athletes but are highly dependent on the severity of injury, as well as the demands and position in sport. Real-world strategies for staged successful rehabilitation are presented and discussed.

$\mathbf{U}$ pper extremity sports injuries are exceedingly common and can have a large impact on athletic participation and performance. It is estimated that almost one third of shoulder injuries are sports related, with a high proportion in young males. ${ }^{1}$ In a recent systematic review, the estimated incidence of shoulder pain in sports is high: up to $32 \%$ for baseball, $47 \%$ for basketball, $63 \%$ for handball, and $52 \%$ for volleyball. ${ }^{2}$ Overhead and throwing athletes place an especially large stress on their shoulders and elbows, with professional pitchers having 58 activity-related shoulder injuries per 100 players annually. ${ }^{3}$ In recovering from these upper extremity injuries, proper postoperative

From the Department of Orthopaedic Surgery, University of Michigan (S.R.O., A.B.), Ann Arbor, Michigan; Midwest Orthopaedics at Rush (N.N.V., L.M.F., N.S.C.), Chicago, Illinois; Department of Orthopaedic Surgery, Rush University Medical Center (N.N.V., L.M.F., N.S.C.), Chicago, Illinois; the Chicago White Sox (B.K., M.M.R.), Chicago, Illinois; and Champion Physical Therapy o Performance (M.M.R.), Waltham, Massachusetts, U.S.A.

This study was performed internally at Rush University Medical Center.

The authors report that they have no conflicts of interest in the authorship and publication of this article. Full ICMJE author disclosure forms are available for this article online, as supplementary material.

Received September 13,2021; accepted September 29, 2021.

Address correspondence to Benjamin Kerzner, Department of Orthopaedic Surgery, Rush University Medical Center, 1611 W Harrison St. Suite 300, Chicago, IL 60612.E-mail: benkerzner@gmail.com

(C) 2021 THE AUTHORS. Published by Elsevier Inc. on behalf of the Arthroscopy Association of North America. This is an open access article under the CC BY-NC-ND license (http://creativecommons.org/licenses/by-nc-nd/4.0/). 2666-061X/211317

https://doi.org/10.1016/j.asmr.2021.09.033 rehabilitation and setting reasonable expectations regarding return to play are very important.

This review article focuses on the most common surgically treated shoulder and elbow injuries, including rotator cuff tears, SLAP tears, anterior and posterior shoulder instability, and elbow ulnar collateral ligament (UCL) tears. This article will aim to summarize the overhead athlete rehabilitation process after surgery and discuss return-to-play (RTP) criteria for athletes after surgery.

\section{General Shoulder Rehabilitation Progression}

The rehabilitation process after shoulder surgery in the overhead athlete will vary depending on the specific injury and surgery performed. In addition to eliminating pain and inflammation, the rehabilitation process for throwing athletes must include the restoration of motion, strength, and endurance, as well as restoration of proprioception, dynamic stability, and neuromuscular control. As the athlete progresses in rehabilitation, sport-specific drills are added to prepare for a gradual return to competition. Neuromuscular control drills are performed throughout recovery and are advanced to provide a continuous challenge to the neuromuscular control system. Proper communication between the surgical and rehabilitation teams must be used to determine specific timelines. Regardless of specific injury, several general principles must be followed with 4 phases: the acute phase, the intermediate 
phase, the advanced strengthening phase, and the return to activity phase (Table 1).

\section{Acute Phase}

The acute phase of rehabilitation begins immediately after surgery and is characterized by a period of predominantly rest and protection. The duration of the acute phase is dependent on the healing constraints of the involved tissues. Range of motion (ROM) exercises are performed immediately in a restricted range beginning with gentle passive and active-assisted ROM. The rehabilitation specialist should be aware that full glenohumeral ROM is often asymmetric in the throwing athlete. These asymmetries are partly due to bony adaptations in the dominant arm. The dominant humerus often has greater retrotorsion, which allows greater glenohumeral external rotation (ER) while decreasing internal rotation (IR). ${ }^{4}$ Thus comparing total arc of rotational motion (TRM) between shoulders is necessary to assess ROM deficits. TRM is the sum of glenohumeral ER and IR when measured at $90^{\circ}$ elevation in the scapular plane. ${ }^{5}$ TRM should be equal between shoulders, although the rotational arc is shifted into ER in the dominant shoulder. During this initial protection phase, passive ROM in external rotation and the plane of the scapula is initiated within limits dictated by the surgical procedure to prevent significant capsular contracture.

Deficits in shoulder TRM and pathological loss of ROM can be the result of soft tissue adaptations. Soft tissue and flexibility exercises for the posterior shoulder musculature are performed early in the rehabilitation process to address these deficits. The posterior shoulder is subjected to extreme repetitive eccentric contractions during throwing, which may result in soft tissue adaptations and loss of IR ROM. ${ }^{6,7}$ Thus this loss of IR is likely due to posterior muscular stiffness, not posterior capsule tightness. ${ }^{8}$ Conversely, it appears that most throwers exhibit significant posterior laxity when evaluated. ${ }^{9,10}$ Common techniques performed include soft tissue mobilization of the posterior musculature (Fig 1), horizontal adduction across the body, and IR stretching at $90^{\circ}$ of abduction.

The rehabilitation specialist should assess the resting position and mobility of the scapula. Frequently, throwers exhibit a posture of rounded shoulders and a forward head. This posture is associated with muscle weakness of the scapular retractors and deep neck flexor muscles because of prolonged elongation or sustained stretches. ${ }^{11,12}$ In addition, the scapula may appear protracted and anteriorly tilted. Increased anterior tilt of the scapula has been associated with a loss of glenohumeral IR in throwers. ${ }^{13,14}$ Muscle weakness may result in improper mechanics or shoulder symptoms. Stretching, soft tissue mobilization, deep tissue lengthening, muscle-energy, and other manual techniques may be needed in these athletes.
Depending on the severity of the injury, strengthening can be performed in the acute phase and often begins with submaximal, pain-free isometrics for all shoulder and scapular movements. Isometrics should be performed at multiple angles throughout the available ROM, with particular emphasis on contraction at the end of the available ROM. Manual rhythmic stabilization drills are performed for internal and external rotators with the arm in the scapular plane at $30^{\circ}$ and $90^{\circ}$ of abduction (Fig 2). Alternating isometric contractions facilitate co-contraction of the anterior and posterior rotator cuff musculature. Rhythmic stabilization drills may also be performed with the patient supine and arm elevated to approximately $90^{\circ}$ to $100^{\circ}$ and $10^{\circ}$ of horizontal abduction (Fig 3). This position is chosen for the initiation of these drills because of the combined centralized line of action of both the rotator cuff and deltoid musculature, generating a humeral head compressive force during muscle contraction. ${ }^{15,16}$ The rehabilitation specialist uses alternating isometric contractions in the flexion, extension, horizontal abduction, and horizontal adduction planes of motion.

Active ROM activities are permitted when adequate muscle strength and balance have been achieved. With the athlete's eyes closed, the rehabilitation specialist passively moves the upper extremity in the planes of flexion, ER, and IR, pauses, and then returns the extremity to the starting position. The patient is then instructed to actively reposition the upper extremity to the previous location. The rehabilitation specialist may perform these joint repositioning activities throughout the available ROM.

Basic closed kinetic chain exercises are also performed during the acute phase. Exercises are initially performed below shoulder level. The athlete may perform weight shifts in the anterior/posterior and medial/ lateral directions. Rhythmic stabilizations may also be performed during weight shifting. As the athlete progresses, a medium-sized ball may be placed on the table, and weight shifts may be performed on the ball. Load bearing exercises can be advanced from the table to the quadruped position. Criteria to progress to the intermediate phase included minimal pain or inflammation and normalized TRM.

\section{Intermediate Phase}

The intermediate phase begins once the athlete has regained near-normal passive ROM and sufficient shoulder strength balance. Any immobilization is discontinued. Lower extremity, core, and trunk strength and stability are critical to efficiently perform overhead activities by transferring and dissipating forces in a coordinated fashion. Therefore full lower extremity strengthening and core stabilization activities are also performed during the intermediate phase. Emphasis will now be placed on regaining proprioception, 
Table 1. Treatment Guidelines for the Overhead Athlete

Phase I-Acute Phase

Goals

Diminish pain and inflammation

Improve posterior soft tissue flexibility

Re-establish posterior strength $\delta$ dynamic stability (muscular balance)

Control functional stresses/strains

Treatment

Abstain from throwing until pain-free full ROM and full strength - specific time determined by physician

Modalities

Electrical stimulation $\&$ cryotherapy as needed

Flexibility

Improve ER and IR ROM at $90^{\circ}$ abduction to normal TRM values

Enhance horizontal adduction flexibility

Gradually stretch into ER and flexion-Do not force into painful ER

Exercises

Rotator cuff strengthening (especially ER) with light-moderate weight

Tubing ER/IR

Side ER

Scapular strengthening exercises

Retractors

Depressors

Protractors

Manual strengthening exercises

Side ER

Supine ER at $45^{\circ}$ of abduction

Side-lying row

Side flexion in the scapular plane

Dynamic rhythmic stabilization exercises

Proprioception training

Electrical stimulation to posterior cuff as needed during exercises

Closed kinetic chain exercises

Maintain core, lower body, and conditioning throughout

Maintain elbow, wrist, \& forearm strength

Criteria to progress to Phase II

Minimal pain or inflammation

Normalized TRM

Baseline muscular strength without fatigue

Phase II-Intermediate Phase

Goals

Progress strengthening exercises

Restore muscular balance (ER/IR)

Enhance dynamic stability

Maintain flexibility and mobility

Improve core stabilization $\&$ lower body strength

Flexibility

Controlled soft tissue mobility and stretching

Especially for IR and horizontal adduction

Gradually restore full ER

Exercises

Progress strengthening exercises

Full rotator cuff and scapula shoulder isotonic program-begin to advance weight

Initiate dynamic stabilization program

Side ER with RS

ER tubing with end range RS

Wall stabilization onto ball

Push-ups onto ball with stabilization

May initiate two-hand plyometric throws Chest pass
Table 1. Continued

Side-to-side

Overhead soccer throws

Criteria to Progress to Phase III

Full, pain-free ROM

Full 5/5 strength with no fatigue

Phase III-Advanced Strengthening Phase

Goals

Aggressive strengthening program

Progress neuromuscular control

Improve strength, power and endurance

Initiate light pre-throwing activities

Exercises

Stretch prior to exercise program-maintain TRM

Continue strengthening program above

Reinitiate upper body program

Dynamic stabilization drills

ER tubing with end range RS at $90^{\circ}$ abduction

Wall stabs in $90^{\circ}$ of abduction and $90^{\circ}$ of ER

Wall dribble with RS in $90^{\circ}$ of abduction $\& 90^{\circ}$ of ER

Plyometrics

Two-hand drills

One-hand drills (90/90 throws, deceleration throws, throw into bounce-back)

Stretch post-exercise

Criteria to Progress to Phase IV

Full ROM and strength

Adequate dynamic stability

Appropriate rehabilitation progression to this point

Phase IV-Return to Activity Phase

Goals

Progress to throwing program

Continue strengthening and flexibility exercises

Return to competitive throwing

Exercises

Mobility and flexibility drills

Shoulder program

Plyometric program

Dynamic stabilization drills

Progress to interval throwing program

Gradually progress to competitive throwing as tolerated

ROM, range of motion; ER, external rotation; IR, internal rotation; TRM, total rotational motion; RS, rhythmic stabilization.

kinesthesia, and dynamic stabilization throughout the athlete's full ROM, particularly at end range. The goals of the intermediate phase are to enhance functional dynamic stability, re-establish neuromuscular control, restore muscular strength and balance, and regain full ROM for throwing.

During this phase, the rehabilitation program progresses to isotonic strengthening activities with emphasis on restoration of muscle balance. Selective muscle activation is also used to restore muscle balance and symmetry. The shoulder external rotator muscles and scapular retractor, protractor and depressor muscles are isolated through a fundamental exercise program for the overhead thrower. ${ }^{11,17-19}$ This exercise program is based on the collective information derived from electromyographic research of numerous investigators. ${ }^{11,20-26}$ These patients 


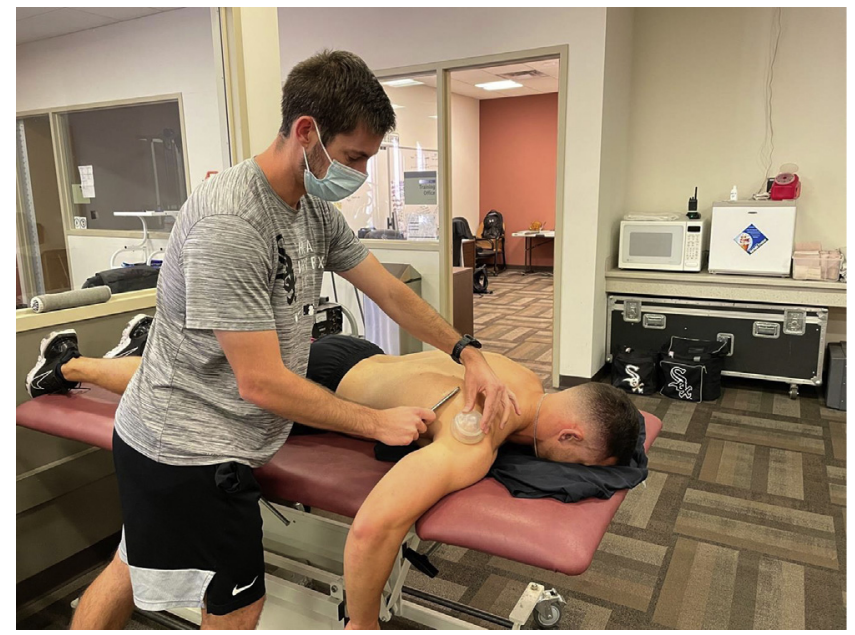

Fig 1. Soft tissue manual therapy for the posterior musculature of the shoulder.

frequently exhibit ER weakness and benefit from side lying ER and prone rowing into ER. Both exercises elicit high levels of muscular activity in the posterior cuff muscles. ${ }^{24}$

Drills performed in the acute phase may be progressed to include stabilization at end ranges of motion with the patient's eyes closed. Rhythmic stabilization exercises are performed during the early part of the intermediate phase. Proprioceptive neuromuscular facilitation exercises are performed in the athlete's available ROM and progressed to include full arcs of motion. Rhythmic stabilizations may be incorporated in various degrees of elevation during the proprioceptive neuromuscular facilitation patterns to promote dynamic stabilization.

Manual resistance external rotation is also performed during the intermediate phase. By applying manual resistance during specific exercises, the rehabilitation specialist can vary the amount of resistance throughout the ROM and incorporate concentric and eccentric contractions, as well as rhythmic stabilizations at end range (Fig 4). As the athlete regains strength and neuromuscular control, ER and IR with tubing may be performed at $90^{\circ}$ of abduction.

Closed kinetic chain exercises are advanced during the intermediate phase. Weight shifting on a ball progresses to a push-up on a ball or unstable surface on a tabletop. Rhythmic stabilizations of the upper extremity, uninvolved shoulder, and trunk are performed with the rehabilitation specialist (Fig 5). Wall stabilization drills can be performed with the athlete's hand on a small ball (Fig 6). Additional axial compression exercises include table and quadruped using a towel around the hand, slide board, or unstable surface. Criteria to enter the advanced phase includes full pain-free ROM and full strength with no fatigue.

\section{Advanced Phase}

The third phase of the rehabilitation program prepares the athlete to return to athletic activity. Full ROM and posterior shoulder muscle flexibility should be maintained throughout this phase. Progressive strengthening of the upper body is also be initiated depending on the needs of the individual patient. Strength needs should be assessed objectively, that is, through handheld or isokinetic dynamometry, because shoulder strength deficits have been prospectively linked with throwing arm injuries. ${ }^{27}$ Exercises such as IR and ER with exercise tubing at $90^{\circ}$ of abduction should be progressed to incorporate eccentric and highspeed contractions.

Plyometrics for the upper extremity may be initiated during this phase to train the upper extremity to dissipate forces. The chest pass, overhead throw, and alternating side-to-side throw with a 4- to 8-pound medicine ball are initially performed with 2 hands. Two-hand drills are progressed to l-hand drills over 10 to 14 days. One-hand plyometrics include baseball style throws in the $90 / 90$ position with a 1- to 2 -pound ball, reverse throws, and stationary and semicircle wall dribbles. They are beneficial for upper extremity endurance while overhead.

Dynamic stabilization and neuromuscular control drills should be reactive, functional, and in sportspecific positions. Concentric and eccentric manual resistance may be applied as the athlete performs external rotation with exercise tubing with the arm at $0^{\circ}$ abduction. Rhythmic stabilizations may be included at end range to challenge the athlete to stabilize against the force of the tubing and progressed to the 90/90 position (Fig 7). Rhythmic stabilizations may be applied at end range during the $90 / 90$ wall dribble exercise. These drills are designed to impart a sudden

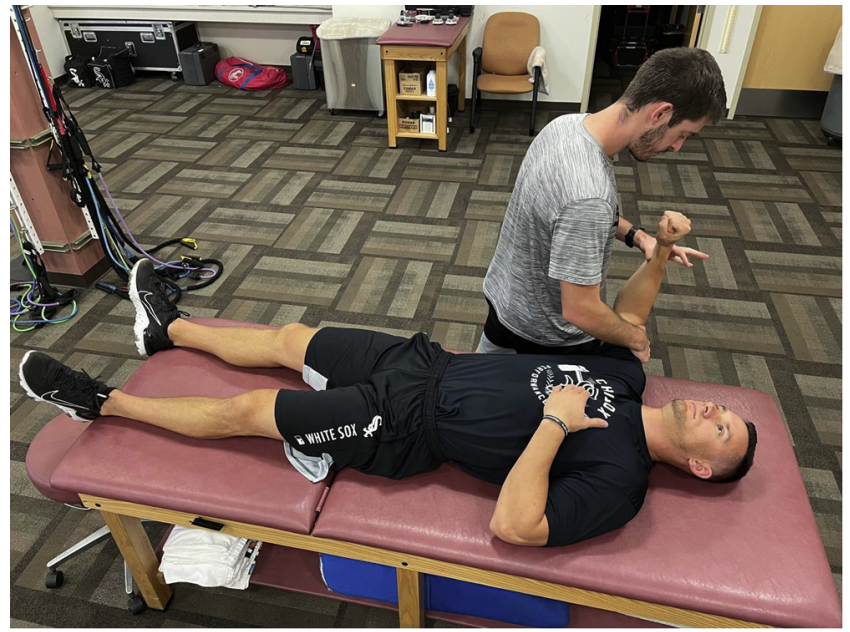

Fig 2. Rhythmic stabilization drills for internal and external rotation with the arm at $90^{\circ}$ of abduction and neutral rotation. 


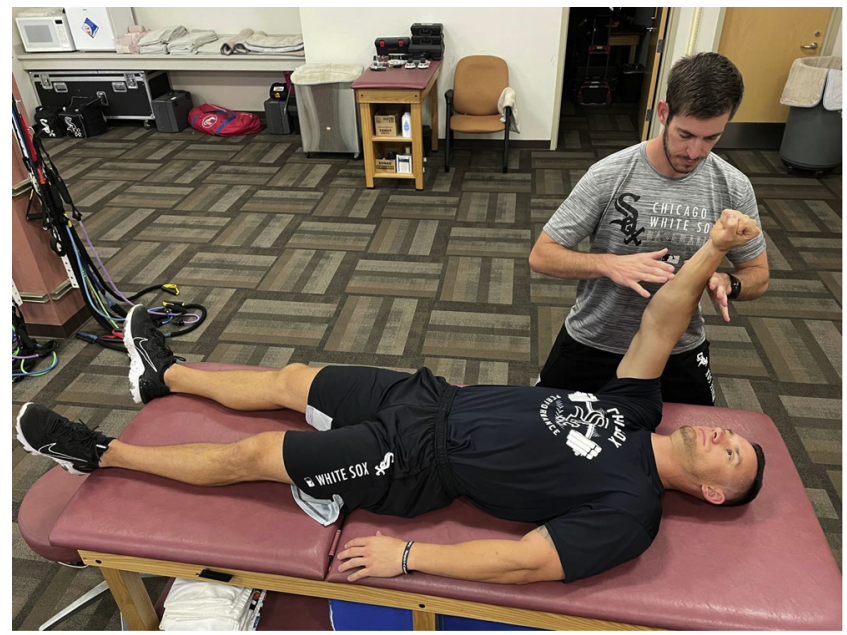

Fig 3. Rhythmic stabilization drills for flexion and extension with the arm elevated to $100^{\circ}$ of flexion in the scapular plane.

perturbation to the throwing shoulder near end range to develop the athlete's ability to dynamically stabilize the shoulder.

Muscle endurance exercises should be emphasized because the overhead athlete is at greater risk for shoulder or elbow injuries when fatigued. ${ }^{28}$ Endurance drills include wall dribbling, ball flips, wall arm circles, or isotonic exercises using lower weights for higher repetitions. The predisposing factor that correlated best with shoulder injuries in Little League pitchers was muscle fatigue. ${ }^{28}$ Thus endurance drills appear critical for the overhead thrower. Criteria to move to the next phase include minimal pain or tenderness, full ROM and strength, adequate proprioception, and dynamic stabilization.

\section{Return-to-Activity Phase}

The previously mentioned strengthening and neuromuscular training program of the shoulder and total body is maintained and gradually progressed during this phase, with the addition of an interval throwing program, which starts with a long toss program designed to gradually increase distance and number of throws. ${ }^{29}$ Athletes typically begin at 30 to 45 feet and gradually progress to 120 feet. The number of throws, distance, and intensity all gradually increase through this phase. Pitchers begin a mound throwing, whereas positional players progress to greater distances of long toss and positional drills. Throwing off the mound includes a gradual increase in the number and intensity of effort, and finally type of pitch. The duration of a throwing program and rate of progression is individualized depending on the injury and nature of a surgical procedure. Although return to play is not evidence based, the general guidelines for decision making for athletes include a holistic approach assessing duration after surgery, ROM progress, overall strength, and their supervised performance program.

\section{Rotator Cuff Repair}

\section{Rehabilitation Timeline}

Rehabilitation protocols after rotator cuff repair vary throughout the current literature, with a paucity of evidence-based guidelines. Many rehabilitation protocols after rotator cuff repair recommend a period of 3 to 4 weeks of immobilization in a sling postoperatively. ${ }^{30-41}$ However duration of immobilization is controversial and some studies indicate early mobilization can be associated with improved outcomes. ${ }^{42}$ During this phase, there is significant heterogeneity in the level of movement recommended by authors in the literature with varying degrees of passive mobilization of the shoulder. ${ }^{30,35,40,41,43,44}$ Athletes will progress through the rehabilitation protocol; further ROM and muscular strengthening will progress between 6 to 12 weeks after surgery and progression to sport specific activities will range from 3 to 6 months. ${ }^{30,31,33,35,43-46}$

\section{Return to Play}

Return to play is permitted after the athlete regains full pain-free shoulder ROM, preinjury shoulder strength $^{38,39,43}$ and clinical/sport-specific testing. ${ }^{30}$ Percentage of players returning to play after rotator cuff repair varies based on the level of athlete, severity of injury, and the sport-specific activity and demand. Elite overhead athletes more dependent on optimal shoulder function had lower RTP after surgery. In a study of 21 professional baseball players, Erickson et al reported only $33.3 \%$ of players were able to RTP. Overall, $14.3 \%$ of these players returned to a same or higher level, but $19 \%$ returned at a lower level. ${ }^{47}$ When performing a mini-open rotator cuff repair on professional pitchers, only 1 of $12(8 \%)$ was able to return to a competitive level of baseball. ${ }^{48}$

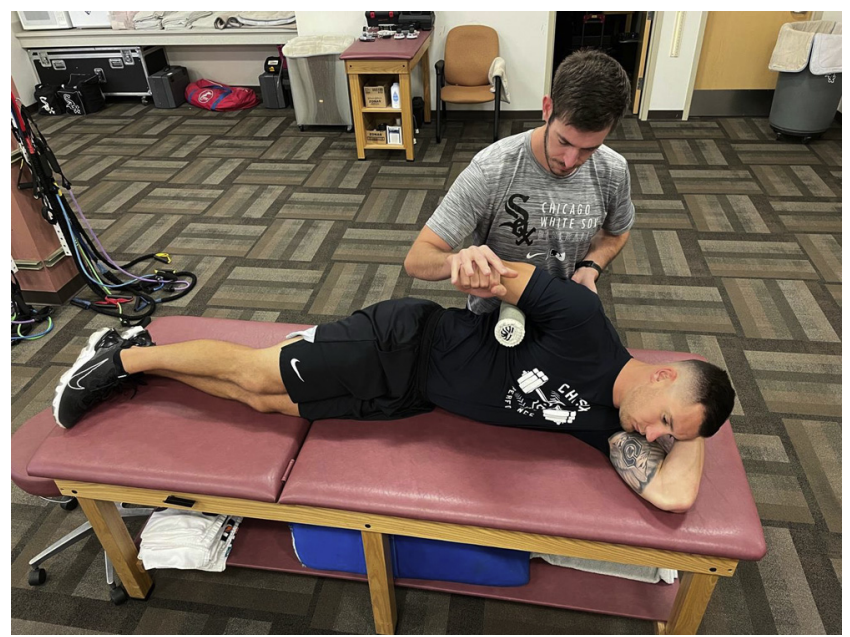

Fig 4. Manual resistance side-lying external rotation with end range rhythmic stabilizations. 


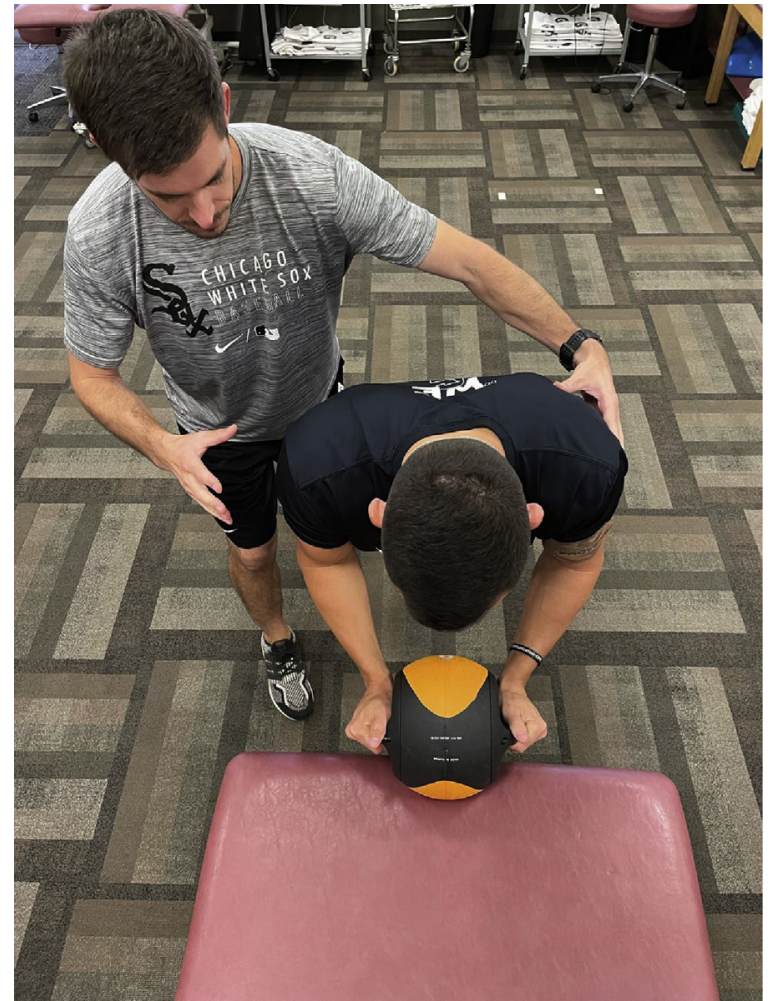

Fig 5. Push-ups on an unstable surface with rhythmic stabilizations applied to the arm and trunk.

In contrast, nonoverhead athletes and recreational athletes were able to RTP after rotator cuff repair more frequently than overhead throwers. In a study of 33 middle-aged and elderly swimmers, Shimada et al. ${ }^{40}$ found that $97 \%$ were able to return to swimming at a mean of 8 months after surgery. In the study of 22 CrossFit athletes, $100 \%$ of patients returned to training after a mean of 8.7 months. ${ }^{32}$ A study of 12 rock climbers undergoing rotator cuff repair showed all 12 returned, but 7 of 12 did not regain their initial climbing level. ${ }^{41}$ A retrospective study of 76 recreational athletes found that $88 \%$ were able to return to sports activity at a similar level a mean of 6 months after surgery. ${ }^{31}$ Athletes younger than 30 years of age who underwent rotator cuff repair showed a high RTP of $85 \%$ a mean of 5.8 months after surgery. ${ }^{33}$

Rates of returning to play are also encouraging in the adolescent patient, and for athletes with partial rotator cuff tears. A study of 27 adolescent athletes with an average age of 16 years who underwent rotator cuff repair found that $93 \%$ were able to return to sport at the same level or higher. ${ }^{43}$ However, 9 of the 14 athletes $(64 \%)$ who played baseball or softball had to switch positions because of a loss of throwing velocity or distance. Another study of adolescent athletes by Weiss et al. ${ }^{49}$ showed $6 / 7$ patients were able to return to their pre-injury level of sport. Studying repair of partial rotator cuff tears, Rossi et al. ${ }^{38}$ found that $87 \%$ of patients $(61 / 72)$ were able to return to sports and 56 patients $(80 \%)$ returned to same level they had before injury. Overhead athletes took significantly longer to return, with a mean time of 6.4 months versus 3.6 months for noncollision, nonoverhead athletes. Last, Klouche et al. ${ }^{50}$ performed a meta-analysis including 25 studies and 859 patients and found that the overall rate of RTP is $84.7 \%$, with $65.9 \%$ returning to preinjury level of play after a range of 4 to 17 months.

\section{SLAP Tears}

\section{Rehabilitation Timeline}

Postoperative therapy protocols after SLAP repair, like the general rehabilitation protocol, have heterogeneity in timelines for progression in the literature. ${ }^{51-56}$ Neri et al. ${ }^{55}$ immobilized overhead athletes' shoulders for 7 to 10 days semioperatively, with active-assisted exercises initiated at 4 weeks. Rotator cuff and periscapular strengthening was started at 6 weeks, and an individualized throwing program was initiated at 12 to 16 weeks. Brockmeier et al. ${ }^{52}$ kept patients in a sling for the first 4 to 6 weeks after surgery and then progressed to functional exercises, proprioceptive training, and sport-specific exercises gradually over 8,12 , and 16 weeks, respectively.

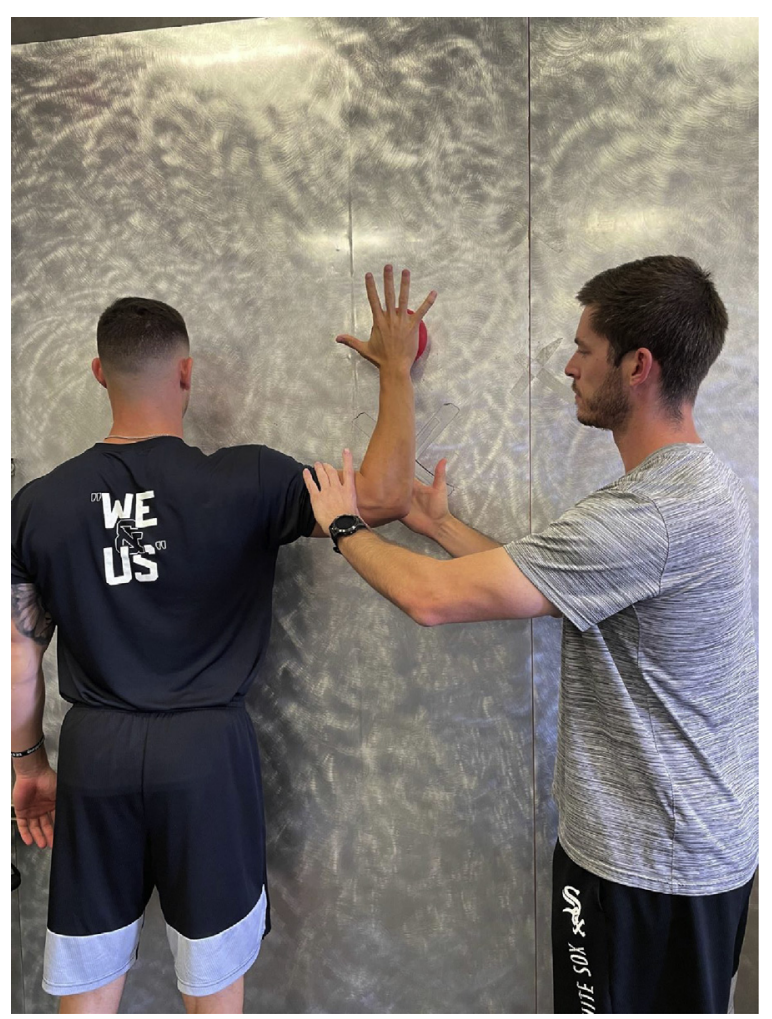

Fig 6. Rhythmic stabilization drills in $90^{\circ}$ of abduction and $90^{\circ}$ of external rotation on an unstable surface in the closed kinetic chain position against the wall. 


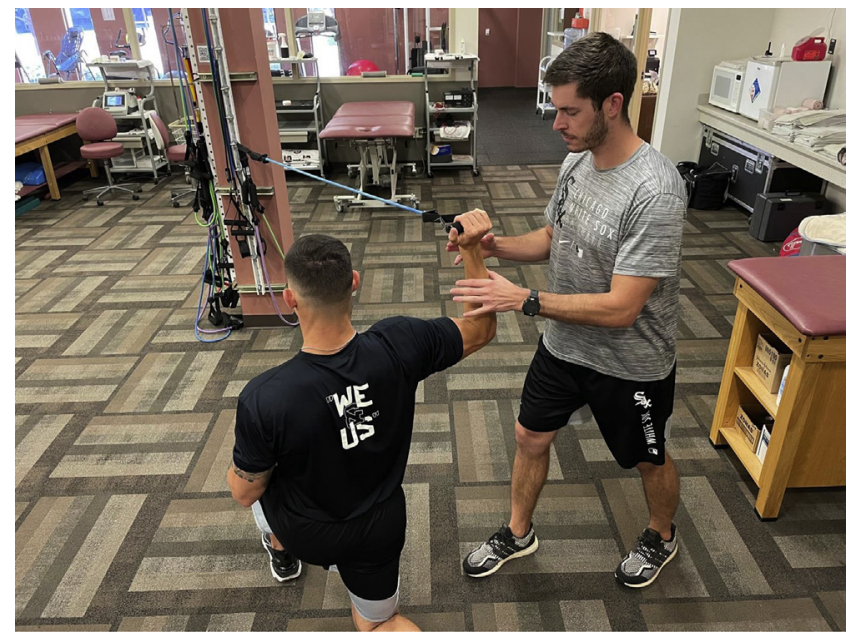

Fig 7. Rhythmic stabilization drills during exercise tubing at $90^{\circ}$ of abduction and $90^{\circ}$ of external rotation.

\section{Return to Play}

Elite overhead throwers have a modest rate of return to play following arthroscopic SLAP repair. Gilliam et al. $^{57}$ looked at 133 male baseball players who required arthroscopic SLAP repair. After a return to throwing protocol, only $41 \%$ of pitchers felt that they were able to return to the same or better level at most recent follow up compared to $64 \%$ of non-pitchers. Seventy-three percent of the pitchers who were unable to return to play indicated that the reason was due to undergoing surgery, whereas $38 \%$ of nonpitchers who were unable to return attributed their situation to undergoing surgery. In another study of 68 major and minor league baseball players that underwent SLAP repair, only 18 of $45(40 \%)$ of pitchers were able to RTP, with only $22 \%$ returning to prior performance. ${ }^{53}$ Van Kleunen et al. ${ }^{58}$ examined 17 baseball players (13 collegiate and 4 high school) who had surgical treatment of an infraspinatus tear and SLAP lesion. Only 6 patients $(35 \%)$ returned to their preinjury level or better, $5(29 \%)$ were participating at a lower level at the same position or had to switch positions because of a decline in speed of throw, and the remaining $6(35 \%)$ patients were unable to return to play.

Return to play rates with non-throwing dependent athletes and mixed athletes are more favorable. A study of 34 professional athletes (including volleyball, football, basketball, tennis) showed $88 \%(30 / 34)$ returned to their preinjury levels with a mean return to play time of 6.4 month. ${ }^{51}$ Brockmeier et al. ${ }^{52}$ studied 47 patients ranging from professional to recreational playing a variety of sports and found that $74 \%$ of the athletes were able to return to their pre-injury level of competition. Friel et al. ${ }^{56}$ noted no statistically significant differences in postoperative subjective functional scores, ROM, and strength among various levels of athletic activity, suggesting that SLAP repair and rehabilitation may be independent of profession, sport, or competition level.

Recently, biceps tenodesis in younger athletes has been shown to be a promising alternative to SLAP repair for SLAP tears. Hurley et al. ${ }^{59}$ performed a retrospective study comparing isolated biceps tenodesis versus SLAP repair in patients younger than 30 years of age. They found statistically similar rates of RTP overall (76\% for tenodesis vs $85 \%$ for repair), time to RTP ( 8.8 months for tenodesis vs 9.4 months for repair), and rate of RTP in overhead athletes $(84 \%$ for tenodesis vs $83 \%$ for repair). A recent systematic review on biceps tenodesis to treat SLAP tears in overhead athletes found an overall RTP rate of $70 \%$, a high American Shoulder and Elbow Surgeons score ranging from 81.7 to 97, and an athlete satisfaction from $80 \%$ to $87 \% .{ }^{60}$

\section{Anterior and Posterior Shoulder Instability}

\section{Rehabilitation Timeline}

Rehabilitation after surgical intervention for shoulder instability can be broadly characterized by a few main phases-protection, endurance, strength, then return to sport. ${ }^{61-65}$ Sling immobilization semioperatively varies significantly across the literature from 0 to 2 weeks, ${ }^{65,66}$ 3 weeks, ${ }^{62} 4$ weeks, ${ }^{61,67}$ or even up to 6 weeks. ${ }^{68}$ After immobilization, athletes can begin active-assisted and active ROM exercises around 4 to 6 weeks semioperatively. ${ }^{63-65,67,69,70}$ Between 6 to 12 weeks semioperatively, strengthening and gradual progression to sport-specific exercises is initiated ${ }^{61,64,65,67-69,71}$ and can extend to $18+$ weeks semioperatively. ${ }^{63}$

\section{Return to Play}

Criteria for return to sports participation includes having a full functional ROM, satisfactory muscular strength and endurance, adequate static and dynamic stability, and clinical examination free of pain. ${ }^{65}$ Athletes typically target return to sports at around 6 to 8 months after surgery. ${ }^{65,67,68,70,72}$

Success in return to play after surgical treatment for anterior and posterior shoulder instability is also variable depending on the sport, position, and nature and severity of injury. In 66 collision athletes, the return to play rate after arthroscopic Bankart repair was $90.9 \%$ at a mean of 6 months. ${ }^{62}$ A study of martial art athletes found 95\% RTP rate in 6.4 months after arthroscopic Bankart repair. ${ }^{67}$ After arthroscopic Bankart repair or open Latarjet reconstruction, 22 of 23 professional athletes were able to return a mean of 4.5 months after surgery. ${ }^{69}$ For posterior shoulder instability, arthroscopic posterior capsulolabral repair in 56 American football players was successful in returning $93 \%$ to play. ${ }^{73}$ In a recent systematic review of posterior shoulder stabilization procedures, 25 studies found an overall $62.7 \%$ to $100 \%$ RTP, with higher return to 
preinjury level for collision athletes compared with overhead athletes. $^{72}$

\section{UCL Tear}

\section{Rehabilitation Protocol and Timeline}

There exists significant variability in rehabilitation protocols after UCL reconstruction of the elbow. Despite no standard consensus on an evidence-based protocol, many studies have reviewed the available literature to identify the core principles of recovery. ${ }^{74-77}$ Current principles of UCL reconstruction (UCL-R) can be divided into 4 phases: (1) immediate postoperative, (2) intermediate strengthening, (3) advanced strengthening, and (4) return to activity. ${ }^{75,77}$

The first phase (weeks 0 to 3 ) is directed at protecting the healing graft and gradual restoration of elbow ROM. ${ }^{75,77}$ A systematic review by Lightsey et al. ${ }^{74}$ analyzed 30 rehabilitation protocols after UCL-R and found that most protocols recommend immediate splinting of the elbow at $90^{\circ}$ for approximately 2 weeks, followed by a functional brace to be worn until approximately 4 to 6 weeks semioperatively. Once preinjury ROM is restored and pain has subsided, the second phase (intermediate strengthening) may be initiated. ${ }^{75,78,79}$

The intermediate strengthening phase (weeks 4 to 7 ) involves gradual increases in upper-extremity mobility, muscular strength, and increasing resistance to valgus stress. ${ }^{75,77}$ Once strength of the operative extremity reaches $70 \%$ of the uninjured extremity, the advanced strengthening phase is initiated. ${ }^{75}$

The advanced strengthening phase (weeks 8 to 14) is comprised of increasing muscle strength, durability, and neuromuscular control. ${ }^{75,77}$ Lightsey et al. ${ }^{74}$ described 8 different plyometric exercises that were commonly cited in protocols, including chest passes, side throw close to body, side-to-side throws, side throws, soccer throws, one-hand throws, one-hand wall dribble, and one-hand baseball throws into wall. Athletes may finally transition to the return-to-activity phase once full, painless ROM is tolerated and strength testing is satisfactory. ${ }^{75}$

During the return to activity phase (weeks 14 to 32), an Interval Throwing Program is recommended to safely reintroduce athletes back into overhead throwing through graduated throwing distances. ${ }^{74,75,77}$ Across most protocols, the average initiation of this program was reported to be approximately 16 weeks after surgery. ${ }^{74,76}$ Pitchers will be introduced into practice games as they gradually increase pitch counts to ensure readiness for return to play. ${ }^{75,79,80}$

\section{Return to Play}

A systematic review by Douoguih et al ${ }^{77}$ reports a return to play target of $9-10$ months and return to previous level of play as high as $81 \%$ to $90 \%$. However, additional studies of elite baseball players suggest that this population may require longer periods of rehabilitation and gradual progression of return to minimize risk of reinjury. A systematic review comprised of 1520 pitchers by Coughlin et al. ${ }^{81}$ reported an increased mean time of return to play of 19.8 months, with a return to competition for Major League Baseball pitchers to be a mean 17.3 months. The authors also described a rate of return to any level of pitching between $79 \%$ to $100 \%{ }^{81}$ Anderson et al. ${ }^{76}$ report a rate of return to competition at the preinjury level or higher of $85.7 \%$ at an average of 12.2 months after surgery. In general, there is strong evidence to suggest that a 4phase rehabilitation protocol after UCL-R with milestone-based progression is successful in returning young overhead athletes back to preinjury level of play.

\section{Conclusion}

Shoulder and elbow injuries during athletic participation are very common and may require operative intervention if refractory to a period of rest and rehabilitation. After surgery, a stepwise rehabilitation process is essential to restore shoulder and elbow motion, muscular strength, proprioception, and neuromuscular control. Rates of return to play after surgical intervention are encouraging in most professional and recreational athletes but are highly dependent on the severity of injury, as well as the demands and position in sport. Unfortunately, professional baseball pitchers have a lower rate of return to elite performance after rotator cuff repair and SLAP repair.

\section{References}

1. Enger M, Skjaker SA, Nordsletten L, et al. Sports-related acute shoulder injuries in an urban population. BMJ Open Sport Exerc Med 2019;5(1):e000551.

2. Kraan RBJ, de Nobel D, Eygendaal D, Daams JG, Kuijer PPFM, Maas M. Incidence, prevalence, and risk factors for elbow and shoulder overuse injuries in youth athletes: A systematic review. Transl Sports Med 2019;2: 186-195.

3. Lin DJ, Wong TT, Kazam JK. Shoulder injuries in the overhead-throwing athlete: Epidemiology, mechanisms of injury, and imaging findings. Radiology 2018;286:370-387.

4. Greenberg EM, Fernandez-Fernandez A, Lawrence JT, McClure P. The development of humeral retrotorsion and its relationship to throwing sports. Sports Health 2015;7: 489-496.

5. Wilk KE, Macrina LC, Fleisig GS, et al. Correlation of glenohumeral internal rotation deficit and total rotational motion to shoulder injuries in professional baseball pitchers. Am J Sports Med 201 1;39:329-335.

6. Reinold MM, Gill TJ. Current concepts in the evaluation and treatment of the shoulder in overhead-throwing athletes, part 1: Physical characteristics and clinical examination. Sports Health 2010;2:39-50. 
7. Reinold MM, Wilk KE, Macrina LC, et al. Changes in shoulder and elbow passive range of motion after pitching in professional baseball players. Am J Sports Med 2008;36: 523-527.

8. Bailey LB, Shanley E, Hawkins R, et al. Mechanisms of shoulder range of motion deficits in asymptomatic baseball players. Am J Sports Med 2015;43:2783-2793.

9. Borsa PA, Dover GC, Wilk KE, Reinold MM. Glenohumeral range of motion and stiffness in professional baseball pitchers. Med Sci Sports Exerc 2006;38:21-26.

10. Borsa PA, Wilk KE, Jacobson JA, et al. Correlation of range of motion and glenohumeral translation in professional baseball pitchers. Am J Sports Med 2005;33:1392-1399.

11. Reinold MM, Escamilla RF, Wilk KE. Current concepts in the scientific and clinical rationale behind exercises for glenohumeral and scapulothoracic musculature. J Orthop Sports Phys Ther 2009;39:105-117.

12. Thigpen CARM, Padua DA, Schneider RS, Distefano LJ, Gill TJ. 3-D scapular position and muscle strength are related in professional baseball pitchers. J Athl Train 2008;43(2):S-S49.

13. Borich MR, Bright JM, Lorello DJ, Cieminski CJ, Buisman T, Ludewig PM. Scapular angular positioning at end range internal rotation in cases of glenohumeral internal rotation deficit. J Orthop Sports Phys Ther 2006;36:926-934.

14. Lukasiewicz AC, McClure P, Michener L, Pratt N, Sennett B. Comparison of 3-dimensional scapular position and orientation between subjects with and without shoulder impingement. J Orthop Sports Phys Ther 1999;29: 574-583. discussion 584-586.

15. Poppen NK, Walker PS. Forces at the glenohumeral joint in abduction. Clin Orthop Relat Res 1978;135:165-170.

16. Walker PS, Poppen NK. Biomechanics of the shoulder joint during abduction in the plane of the scapula [proceedings]. Bull Hosp Joint Dis 1977;38:107-111.

17. Wilk KE, Reinold MM, Andrews JR. Rehabilitation of the thrower's elbow. Clin Sports Med 2004;23:765-801. xii.

18. Wilk KE, Reinold MM, Dugas JR, Andrews JR. Rehabilitation following thermal-assisted capsular shrinkage of the glenohumeral joint: Current concepts. J Orthop Sports Phys Ther 2002;32:268-292.

19. Wilk KE, Reinold MM, Dugas JR, Arrigo CA, Moser MW, Andrews JR. Current concepts in the recognition and treatment of superior labral (SLAP) lesions. J Orthop Sports Phys Ther 2005;35:273-291.

20. Blackburn TAMW, White B. Electromyographic analysis of posterior rotator cuff exercises. J Athl Train 1990;25: 40-45.

21. Decker MJ, Hintermeister RA, Faber KJ, Hawkins RJ. Serratus anterior muscle activity during selected rehabilitation exercises. Am J Sports Med 1999;27:784-791.

22. Moseley JB Jr, Jobe FW, Pink M, Perry J, Tibone J. EMG analysis of the scapular muscles during a shoulder rehabilitation program. Am J Sports Med 1992;20:128-134.

23. Reinold MM, Macrina LC, Wilk KE, et al. Electromyographic analysis of the supraspinatus and deltoid muscles during 3 common rehabilitation exercises. J Athl Train 2007;42:464-469.

24. Reinold MM, Wilk KE, Fleisig GS, et al. Electromyographic analysis of the rotator cuff and deltoid musculature during common shoulder external rotation exercises. J Orthop Sports Phys Ther 2004;34: 385-394.

25. Townsend H, Jobe FW, Pink M, Perry J. Electromyographic analysis of the glenohumeral muscles during a baseball rehabilitation program. Am J Sports Med 1991;19: 264-272.

26. Worrell TW, Corey BJ, York SL, Santiestaban J. An analysis of supraspinatus EMG activity and shoulder isometric force development. Med Sci Sports Exerc 1992;24: 744-748.

27. Byram IR, Bushnell BD, Dugger K, Charron K, Harrell FE Jr, Noonan TJ. Preseason shoulder strength measurements in professional baseball pitchers: Identifying players at risk for injury. Am J Sports Med 2010;38: 1375-1382.

28. Lyman S, Fleisig GS, Andrews JR, Osinski ED. Effect of pitch type, pitch count, and pitching mechanics on risk of elbow and shoulder pain in youth baseball pitchers. Am J Sports Med 2002;30:463-468.

29. Reinold MM, Wilk KE, Reed J, Crenshaw K, Andrews JR. Interval sport programs: Guidelines for baseball, tennis, and golf. J Orthop Sports Phys Ther 2002;32:293-298.

30. Altintas B, Bradley H, Logan C, Delvecchio B, Anderson N, Millett PJ. Rehabilitation following subscapularis tendon repair. Int J Sports Phys Ther 2019;14:318-332.

31. Antoni M, Klouche S, Mas V, Ferrand M, Bauer T, Hardy P. Return to recreational sport and clinical outcomes with at least 2 years follow-up after arthroscopic repair of rotator cuff tears. Orthop Traumatol Surg Res 2016;102:563-567.

32. Carbone S, Candela V, Gumina S. High rate of return to CrossFit training after arthroscopic management of rotator cuff tear. Orthop J Sports Med 2020;8:232596 7120911039 .

33. Davey MS, Hurley ET, Scanlon JP, Gaafar M, Pauzenberger L, Mullett H. Excellent clinical outcomes and rates of return to play after arthroscopic rotator cuff repair for traumatic tears in athletes aged 30 years or less. Arthrosc Sports Med Rehabil 2021;3(3):e667-e672.

34. Liem D, Lichtenberg S, Magosch P, Habermeyer P. Arthroscopic rotator cuff repair in overhead-throwing athletes. Am J Sports Med 2008;36:1317-1322.

35. Merolla G, Paladini P, Porcellini G. Assessment of return to play in professional overhead athletes subjected to arthroscopic repair of rotator cuff tears and associated labral injuries using the Italian version of the Kerlan-Jobe Orthopedic Clinic Shoulder and Elbow score. Musculoskelet Surg 2018;102:29-34 (Suppl 1).

36. Mihata T, Lee TQ, Fukunishi K, et al. Return to sports and physical work after arthroscopic superior capsule reconstruction among patients with irreparable rotator cuff tears. Am J Sports Med 2018;46:1077-1083.

37. Mihata T, Lee TQ, Hasegawa A, et al. Five-year follow-up of arthroscopic superior capsule reconstruction for irreparable rotator cuff tears. J Bone Joint Surg Am 2019;101: 1921-1930.

38. Rossi LA, Atala N, Bertona A, et al. Return to sports after in situ arthroscopic repair of partial rotator cuff tears. Arthroscopy 2019;35:32-37. 
39. Rossi LA, Atala NA, Bertona A, et al. Long-term outcomes after in situ arthroscopic repair of partial rotator cuff tears. Arthroscopy 2019;35:698-702.

40. Shimada Y, Sugaya H, Takahashi N, et al. Return to sport after arthroscopic rotator cuff repair in middle-aged and elderly swimmers. Orthop J Sports Med 2020;8(6): 2325967120922203.

41. Simon M, Popp D, Lutter C, Schöffl V. Functional and sports-specific outcome after surgical repair of rotator cuff tears in rock climbers. Wilderness Environ Med 2017;28: 342-347.

42. Houck DA, Kraeutler MJ, Schuette HB, McCarty EC, Bravman JT. Early versus delayed motion after rotator cuff repair: A systematic review of overlapping meta-analyses. Am J Sports Med 2017;45:2911-2915.

43. Azzam MG, Dugas JR, Andrews JR, Goldstein SR, Emblom BA, Cain EL Jr. Rotator cuff repair in adolescent athletes. Am J Sports Med 2018;46:1084-1090.

44. Bhatia S, Greenspoon JA, Horan MP, Warth RJ, Millett PJ. Two-year outcomes after arthroscopic rotator cuff repair in recreational athletes older than 70 years. Am J Sports Med 2015;43:1737-1742.

45. Tambe A, Badge R, Funk L. Arthroscopic rotator cuff repair in elite rugby players. Int J Shoulder Surg 2009;3: 8-12.

46. Weber A, Paraparan R, Lam PH, Murrell GAC. Return to sport at 6 months after shoulder surgery. Orthop J Sports Med 2019;7(3):2325967119834077.

47. Erickson BJ, Chalmers PN, D'Angelo J, Ma K, Romeo AA. Performance and return to sport following rotator cuff surgery in professional baseball players. J Shoulder Elbow Surg 2019;28:2326-2333.

48. Mazoué CG, Andrews JR. Repair of full-thickness rotator cuff tears in professional baseball players. Am J Sports Med 2006;34:182-189.

49. Weiss JM, Arkader A, Wells LM, Ganley TJ. Rotator cuff injuries in adolescent athletes. J Pediatr Orthop B 2013;22: 133-137.

50. Klouche S, Lefevre N, Herman S, Gerometta A, Bohu Y. Return to sport after rotator cuff tear repair: A systematic review and meta-analysis. Am J Sports Med 2016;44: 1877-1887.

51. Beyzadeoglu T, Circi E. Superior labrum anterior posterior lesions and associated injuries: Return to play in elite athletes. Orthop J Sports Med 2015;3(4):23259671155 77359 .

52. Brockmeier SF, Voos JE, Williams RJ 3rd, Altchek DW, Cordasco FA, Allen AA. Outcomes after arthroscopic repair of type-II SLAP lesions. J Bone Joint Surg Am 2009;91:1595-1603.

53. Fedoriw WW, Ramkumar P, McCulloch PC, Lintner DM. Return to play after treatment of superior labral tears in professional baseball players. Am J Sports Med 2014;42: 1155-1160.

54. Neuman BJ, Boisvert CB, Reiter B, Lawson K, Ciccotti MG, Cohen SB. Results of arthroscopic repair of type II superior labral anterior posterior lesions in overhead athletes: Assessment of return to preinjury playing level and satisfaction. Am J Sports Med 201 1;39:1883-1888.

55. Neri BR, ElAttrache NS, Owsley KC, Mohr K, Yocum LA. Outcome of type II superior labral anterior posterior repairs in elite overhead athletes: Effect of concomitant partial-thickness rotator cuff tears. Am J Sports Med Jan $2011 ; 39: 114-120$.

56. Friel NA, Karas V, Slabaugh MA, Cole BJ. Outcomes of type II superior labrum, anterior to posterior (SLAP) repair: Prospective evaluation at a minimum two-year follow-up. J Shoulder Elbow Surg 2010;19:859-867.

57. Gilliam BD, Douglas L, Fleisig GS, et al. Return to play and outcomes in baseball players after superior labral anterior-posterior repairs. Am J Sports Med 2018;46: 109- 115.

58. Van Kleunen JP, Tucker SA, Field LD, Savoie FH 3rd. Return to high-level throwing after combination infraspinatus repair, SLAP repair, and release of glenohumeral internal rotation deficit. Am J Sports Med 2012;40: 2536-2541.

59. Hurley ET, Colasanti CA, Lorentz NA, et al. Open subpectoral biceps tenodesis may be an alternative to arthroscopic repair for SLAP tears in patients under 30 [published online July 31, 2021]. Arthroscopy. doi:10. 1016/j.arthro.2021.07.028.

60. Frantz TL, Shacklett AG, Martin AS, et al. Biceps tenodesis for superior labrum anterior-posterior tear in the overhead athlete: A systematic review. Am J Sports Med 2021;49:522-528.

61. Bradley H, Lacheta L, Goldenberg BT, Rosenberg SI, Provencher MT, Millett PJ. Latarjet procedure for the treatment of anterior glenohumeral instability in the athlete-Key considerations for rehabilitation. Int J Sports Phys Ther 2021;16:259-269.

62. Davey MS, Hurley ET, Colasanti CA, et al. Clinical outcomes of patients with anterior shoulder instability and glenolabral articular disruption lesions: A retrospective comparative study. Am J Sports Med 2020;48:3472-3477.

63. Goldenberg BT, Goldsten P, Lacheta L, Arner JW, Provencher MT, Millett PJ. Rehabilitation following posterior shoulder stabilization. Int J Sports Phys Ther 202 1;16: 930-940.

64. Koczan B, Stryder B, Mitchell C. Postoperative rehabilitation of posterior glenohumeral joint instability surgery: A systematic review. Sports Med Arthrosc Rev 2021;29: 110-118.

65. Ma R, Brimmo OA, Li X, Colbert L. Current concepts in rehabilitation for traumatic anterior shoulder instability. Curr Rev Musculoskelet Med 2017;10:499-506.

66. Mitchell BC, Siow MY, Carroll AN, Pennock AT, Edmonds EW. Clinical outcomes, survivorship, and return to sport after arthroscopic capsular repair with suture anchors for adolescent multidirectional shoulder instability: Results at 6-year follow-up. Orthop J Sports Med 2021;9(2):2325967121993879.

67. Ranalletta M, Rossi LA, Sirio A, et al. Return to sports and recurrences after arthroscopic anterior shoulder stabilization in martial arts athletes. Orthop J Sports Med 2017;5(9):2325967117725031.

68. Eckenrode BJ, Logerstedt DS, Sennett BJ. Rehabilitation and functional outcomes in collegiate wrestlers following a posterior shoulder stabilization procedure. J Orthop Sports Phys Ther 2009;39:550-559.

69. Dekker TJ, Goldenberg B, Lacheta L, PH M, Millett PJ. Anterior shoulder instability in the professional athlete: 
return to competition, time to return, and career length. Orthop J Sports Med 2020;8(11):2325967120959728.

70. McClincy MP, Arner JW, Bradley JP. Posterior shoulder instability in throwing athletes: A case-matched comparison of throwers and non-throwers. Arthroscopy 2015;31: 1041-1051.

71. Colegate-Stone TJ, van der Watt C, de Beer JF. Evaluation of functional outcomes and complications following modified Latarjet reconstruction in athletes with anterior shoulder instability. Shoulder Elbow 2015;7:168-173.

72. Fried JW, Hurley ET, Duenes ML, et al. Return to play after arthroscopic stabilization for posterior shoulder instability-A systematic review. Arthrosc Sports Med Rehabil 2021;3(1):e249-e256.

73. Arner JW, McClincy MP, Bradley JP. Arthroscopic stabilization of posterior shoulder instability is successful in American football players. Arthroscopy 2015;31:1466-1471.

74. Lightsey HM, Trofa DP, Sonnenfeld JJ, Swindell HW, Makhni EC, Ahmad CS. Rehabilitation variability after elbow ulnar collateral ligament reconstruction. Orthop $J$ Sports Med 2019;7:2325967119833363.

75. Bogdanov JM, Bolia IK, Benvegnu N, Michener LA, Weber AE, Petrigliano FA. Rehabilitation following ulnar collateral ligament reconstruction in overhead-throwing athletes. JBJS Rev 2021;9(4).
76. Anderson MJJ, Crockatt WK, Mueller JD, et al. Returnto-Competition Criteria After Ulnar Collateral Ligament Reconstruction: A Systematic Review and Meta-analysis [published online June 28, 2021]. Am J Sports Med. doi:10.1177/03635465211016839.

77. Douguih WA, Day J, Bahrun EA, Bodendorfer BM, Brandt C, Looney A. Postoperative rehabilitation of ulnar collateral ligament reconstruction: A systematic review. Sports Med Arthrosc Rev 2021;29(2):e9-e17.

78. Ellenbecker TS, Wilk KE, Altchek DW, Andrews JR. Current concepts in rehabilitation following ulnar collateral ligament reconstruction. Sports Health 2009;1: 301-313.

79. Wilk K, Arrigo C, Dugas J, Cain EL, Andrews J. Rehabilitation and return-to-play criteria following ulnar collateral ligament reconstruction. Operative Tech Sports Med 2017;25:154-171.

80. Cain EL Jr, McGonigle O. Return to play following ulnar collateral ligament reconstruction. Clin Sports Med 2016;35:577-595.

81. Coughlin RP, Gohal C, Horner NS, et al. Return to play and in-game performance statistics among pitchers after ulnar collateral ligament reconstruction of the elbow: A systematic review. Am J Sports Med 2019;47:2003-2010. 